First publ. in: Physica / B [Condensed Matter], Vols. 249-251 (1998), pp. 640-643

\title{
Phase diagram of the screened two-dimensional electron crystal
}

\author{
G. Mistura ${ }^{a}, b$, S. Neser ${ }^{a}, *, P$. Leiderer ${ }^{a}$ \\ "Fakultät für Physik, Universität Konstanz, D-78434 Konstanz, Germany \\ ${ }^{b}$ Instituto Nazionale per la Fisica della Materia and Dip.to di Fisica, Universitá di Padova, via Marzolo 8. 35131 Padova, Italy
}

\begin{abstract}
The melting line of the two-dimensional crystal formed by surface electrons above liquid helium films adsorbed on PMMA has been mapped out by means of a microwave cavity technique at temperatures above $1 \mathrm{~K}$. The influence of the dielectric substrate on such a transition has been measured at different temperatures and at various helium film thicknesses. The results are found to be in good agreement with existing theories. In general, screening reduces the stability range of the Wigner crystal in the phase diagram of the surface electrons. (C) 1998 Elsevier Science B.V. All rights reserved.
\end{abstract}

Keywords: Wigner solid; Screening; Liquid helium

Surface electrons (SE) formed at the surface of bulk liquid helium constitute a nearly ideal twodimensional electron system (2DES) which is characterized by the presence, in zero magnetic field, of a low density crystalline phase below $1 \mathrm{~K}[1]$. The transition into this classical crystal has been first observed almost 20 years ago in a landmark experiment $[2,3]$. The 2DES is also expected to undergo a quantum melting transition at low $T$ and very high electron density, $n>10^{13} \mathrm{~cm}^{-2}$ [4]. Unfortunately, such a regime cannot be reached on the surface of bulk liquid helium because an electrohydrodynamic instability of the charged surface limits $n$ to values below $2.2 \times 10^{9} \mathrm{~cm}^{-2}$ [1]. In order to increase the maximum density of the SE

*Corresponding author. Tel.: + 49753188 3808; Fax: + 49 753188 3127; e-mail: stephan.neser@uni-konstanz.de. system, it has been suggested to use a thin helium film as a substrate for the SE, because a film is additionally stabilized by van der Waals forces [5]. This has been demonstrated by Etz et al. [6] who were able to charge helium films to electron densities of $\approx 10^{11} \mathrm{~cm}^{-2}$. Furthermore, on a thin helium film, the interparticle interaction is modified due to the screening of the electron-electron interaction by the dielectric substrate supporting the helium film. The major consequence of such a screening - which so far, however, had only been considered theoretically - is the shrinking of the solid phase region with respect to the $2 \mathrm{DES}$ phase diagram on bulk helium [7,8]: the classical freezing line is pushed to higher density and lower temperature, while the quantum melting is shifted to lower $n$ and $T$. Regarding the classical part of the screened 2DES phase diagram, Saitoh [9] has determined the dependence of the melting density $n_{\mathrm{M}}$ of the 
screened crystal on the helium film thickness $d_{\mathrm{He}}$ following the Kosterlitz and Thouless melting criterion. The explicit expression of this formula is [9]:

$k_{\mathrm{B}} T_{\mathrm{M}}\left(d_{\mathrm{He}}\right)=\frac{e^{2} \sqrt{\pi n}}{4 \pi \varepsilon_{0} \varepsilon_{\mathrm{He}} \Gamma}\left[1-\frac{\delta}{\left(1+\left[\left(4 \pi n d_{\mathrm{He}}^{2}\right) / c^{2}\right]\right)^{3 / 2}}\right]$,

where $c=1.1061$ is a constant, $\Gamma$ is the plasma parameter (that is the ratio of the mean potential energy to the mean kinetic energy of one electron), and $\delta=\left(\varepsilon_{\text {sub }}-1\right) /\left(\varepsilon_{\text {sub }}+1\right)$, where $\varepsilon_{\text {sub }}$ is the dielectric constant of the substrate. As for the quantum melting line, there exist in the literature many differing theoretical predictions of its location [10], while there is yet no conclusive experimental evidence of such a transition. A first hint of the crossing of the quantum melting line may have been observed in a microwave cavity experiment of SE on helium films covering $\mathrm{SiO}_{2}$ [11]. .

Hereafter we report the results of the first systematic study of the classical melting line of a screened 2DES formed by SE above helium films covering a poly(methyl-methacrylate) (PMMA) surface. The microwave cavity setup used in this study has been described in detail elsewhere [12]. A thin silicon platelet, $5 \times 25 \mathrm{~mm}$, spin-coated with a thin PMMA layer $\left(d_{\mathrm{s}} \approx 25 \mathrm{~nm}\right)$, was placed along the axis of a cylindrical cavity which was excited in the fundamental $\mathrm{TM}_{010}$ mode at a frequency $f \approx 10 \mathrm{GHz}$ when the sample was inside. An initial helium film of thickness $d_{\mathrm{He}}$ was prepared by keeping the level of liquid helium at a height $H$ below the substrate. The distance $H$ was determined by the frequency shift of the cavity when liquid helium was inside. The helium film was charged by firing a small tungsten filament while increasing the generally positive clamping voltage $U$ applied to the silicon platelet in small steps so that the electrons could not acquire sufficient energy to penetrate into the helium film. The resonator was inserted in a microwave transmission line with a feedback loop which locked the frequency of an X-band generator onto the cavity resonance. The frequency stability was $\approx 3 \mathrm{ppm}$, that of the amplitude $\approx 0.3 \%$. Both the resonance frequency and the transmitted power through the cavity were monitored during charg- ing. The real $\chi$ and imaginary $\alpha$ parts of the dielectric response of the SE were calculated from the resonance frequency and amplitude [12].

Fig. 1 shows three charging runs at two different temperatures and on helium films of two different initial thicknesses covering a PMMA layer approximately $25 \mathrm{~nm}$ thick. On charging the film the absorption rises almost linearly with increasing electron density. The susceptibility is also linear and has a negative value, which means that the charging has led to an increase of the cavity resonance. This is the familiar behavior of free, noninteracting electrons obeying the Drude model where both the absorbed power and susceptibility are simply linear in $n$. As $n$ increases, the SE remain in a fluid phase characterized by an increasing

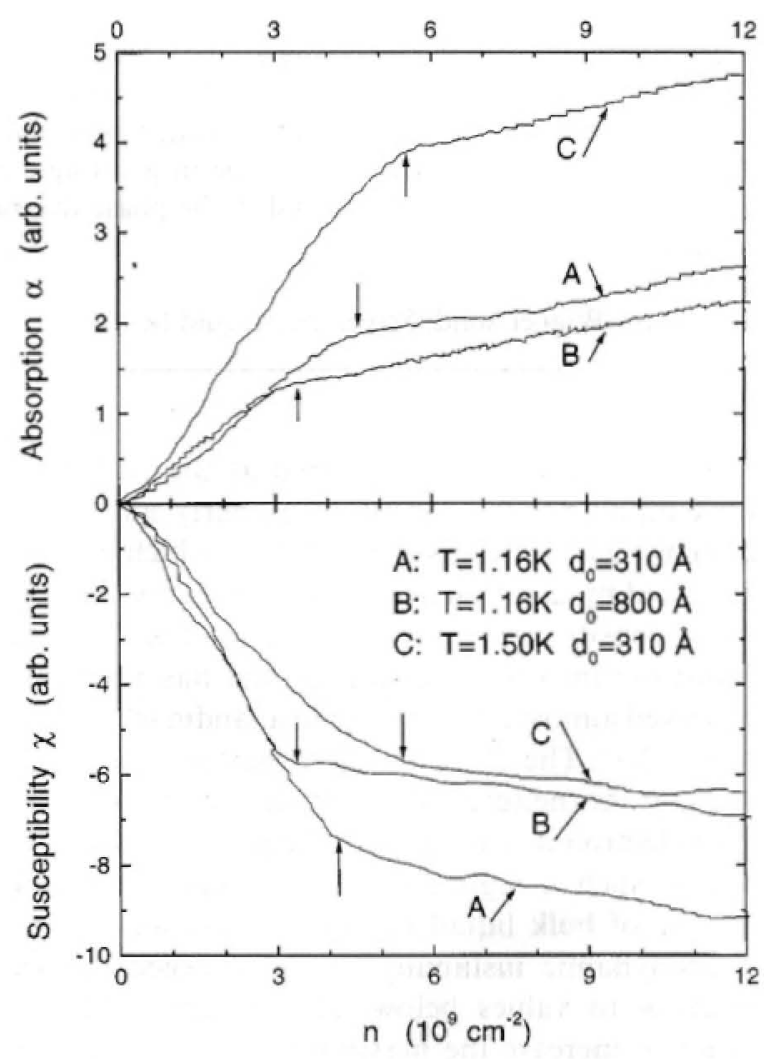

Fig. 1. Relative absorption $\alpha$ and susceptibility $\chi$ of the SE at two different temperatures and on two helium films of different initial thickness covering a PMMA layer versus electron density $n$. 
electronic correlation until a critical value is reached above which there is an abrupt change in the SE mobility as signalled by the simultaneous kinks in both $\alpha$ and $\chi$. We identify this kink as the onset of Wigner crystallization [12,13]. More precisely, the change in the electron absorption at solidification is due to the pinning of the 2DES crystal to some surface defects. In reality, as the 2DES experiences a strong random potential due to surface roughness and pinned electrons, it is more likely that the 2DES freezes in many small crystallites instead of in one large single crystal. Some small crystallites can still move and absorb microwave energy, which explains why we only observed a kink and not a sharp jump down to $\alpha=0$ at solidification.

Fig. 2 shows the dependence of the melting density with temperature in the range $1.15-1.6 \mathrm{~K}$ and on two charged helium film thicknesses of $\approx 300 \AA$ and $\approx 500 \AA$. In the same figure we have also plotted the predictions of Eq. (1) for $D=300 \AA$ and $500 \AA$. The two lines in Fig. 2 represent the fit of the data points using the plasma parameter $\Gamma$ as a free parameter. We find that the best fit to the data is

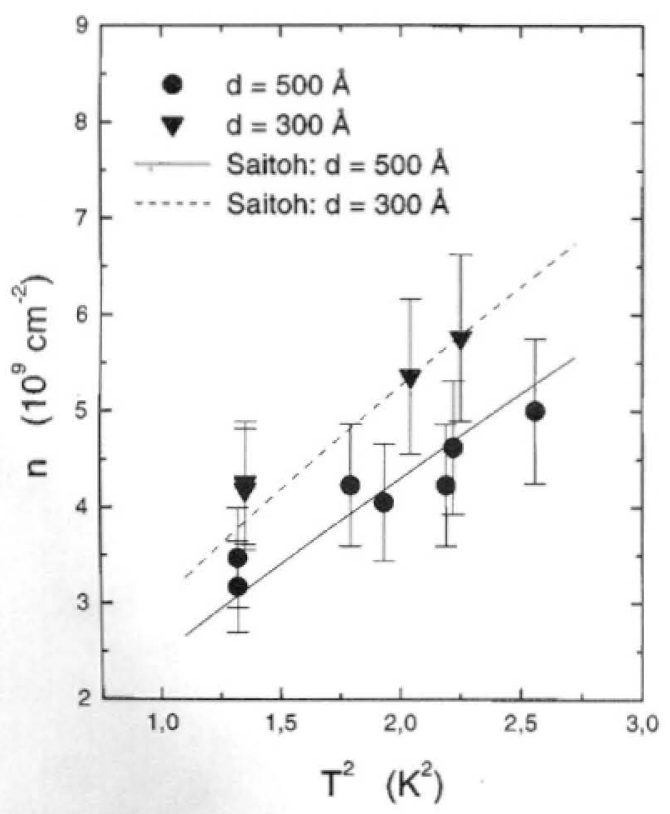

Fig. 2. Melting density $n_{\mathrm{M}}$ of the SE crystal on charged helium films of different initial thickness as a function of the temperature. For further information see text. achieved with $\Gamma=117 \pm 15$, quite close to the value of 127 found for 2DES on bulk helium $[2,3,14]$. Furthermore, Eq. (1) indicates that the melting density of a screened SE crystal also depends on the thickness of the helium film.

In Fig. 3 we report the experimental values of $n_{\mathrm{M}}$ as a function of the charged helium film thickness together with the predictions of Eq. (1) using again $\Gamma$ as a free parameter. The best fit to the data is achieved with $\Gamma=124 \pm 20$, in good agreement with the value deduced from Fig. 1.

In conclusion, the results presented here show that the classical part of the Wigner crystal phase diagram follows the theoretical predictions for screening of the electronic interaction quite closely. As the influence of the polarization of the substrate is increased, the crystallization line is shifted to higher electron densities, as expected from the reduction of the electrostatic energy. It will be interesting to study with the same technique the effect of screening in the high density phase diagram where quantum melting of the Wigner crystal occurs. There, as pointed out in the introduction, screening

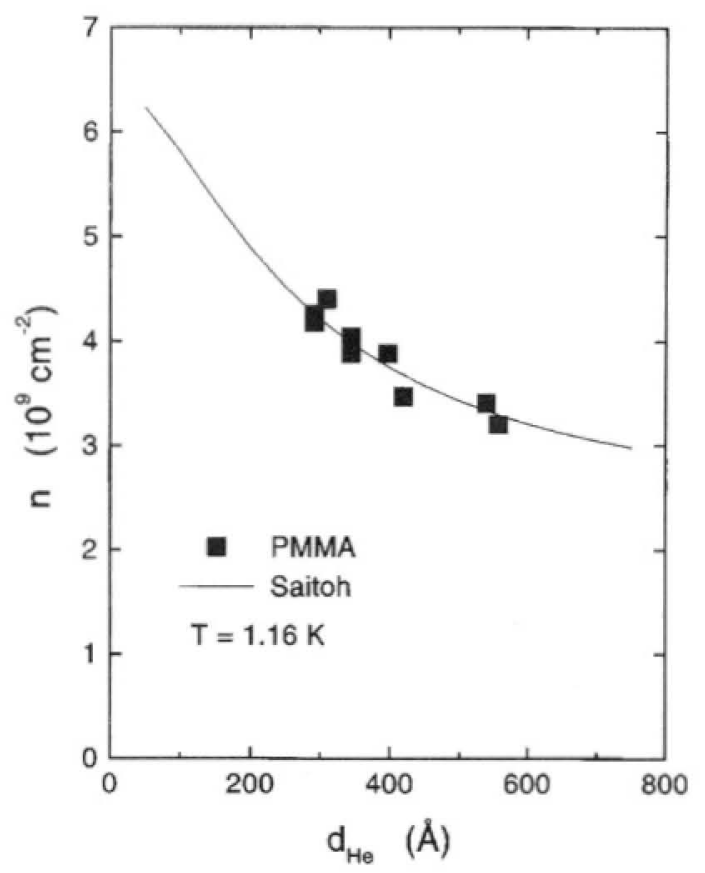

Fig. 3. Melting density $n_{\mathrm{M}}$ of the SE crystal on charged helium films of different initial thickness. For more information see text. 
of the Coulomb potential should lead to a lowering of the melting density. In view of the fact that the details of quantum melting of the 2D Wigner solid are not yet understood, such measurements should provide important information about this phase transition.

We appreciate valuable contributions to the experiment by T. Günzler. This work is supported by the Deutsche Forschungsgemeinschaft and the HCM program of the European Community.

\section{References}

[1] See e.g., in: 2D Electron systems on helium and other cryogenic substrates, E.Y. Andrei (Ed.), Kluwer, Dordrecht, 1997.

[2] C.C. Grimes, G. Adams, Phys. Rev. Lett. 42 (1979) 795.
[3] D.S. Fisher, B.I. Halperin, P.M. Platzmann, Phys. Rev. Lett. 42 (1979) 798.

[4] P.M. Platzman, H. Fukuyama, Phys. Rev. B 10 (1974) 3150.

[5] Yu. P. Monarkha, V.B. Shikin, Fiz. Nizk. Temp. 8 (1982) 563 [Sov. J. Low Temp. Phys, 8 (1982) 279].

[6] H. Etz, W. Gombert, W. Idstein, P. Leiderer, Phys. Rev. Lett. 53 (1984) 2567.

[7] F.M. Peeters, P.M. Platzman, Phys. Rev. Lett. 50 (1983) 2021.

[8] F.M. Peeters, Phys. Rev. B 30 (1984) 159

[9] M. Saitoh, Phys. Rev. B 40 (1989) 810.

[10] See e.g., T. Ando, A.B. Fowler, F. Stern, Rev. Mod. Phys. 54 (1982) 437.

[11] T. Günzler, B. Bitnar, G. Mistura, S. Neser, P. Leiderer, Surf. Sci. 361/362 (1996) 831.

[12] G. Mistura, T. Günzler, S. Neser, P. Leiderer, Phys. Rev. B $56(1997) 8360$.

[13] G. Mistura, O. Tress, E. Teske, V. Shikin, P. Wyder, P. Leiderer, J. Low Temp. Phys, to be published.

[14] G. Deville, J. Low Temp. Phys. 72 (1988) 135. 